\title{
The Least Squares Hermitian (Anti)reflexive Solution with the Least Norm to Matrix Equation $A X B=C$
}

\author{
Xin Liu ${ }^{1}$ and Qing-Wen Wang ${ }^{2}$ \\ ${ }^{1}$ Faculty of Information Technology, Macau University of Science and Technology, Avenida Wai Long, \\ Taipa 999078, Macau, China \\ ${ }^{2}$ Department of Mathematics, Shanghai University, Shanghai 200444, China \\ Correspondence should be addressed to Xin Liu; xiliu@must.edu.mo
}

Received 28 June 2017; Accepted 20 July 2017; Published 29 August 2017

Academic Editor: Kishin Sadarangani

Copyright (C) 2017 Xin Liu and Qing-Wen Wang. This is an open access article distributed under the Creative Commons Attribution License, which permits unrestricted use, distribution, and reproduction in any medium, provided the original work is properly cited.

For a given generalized reflection matrix $J$, that is, $J^{H}=J, J^{2}=I$, where $J^{H}$ is the conjugate transpose matrix of $J$, a matrix $A \in C^{n \times n}$ is called a Hermitian (anti)reflexive matrix with respect to $J$ if $A^{H}=A$ and $A= \pm J A J$. By using the Kronecker product, we derive the explicit expression of least squares Hermitian (anti)reflexive solution with the least norm to matrix equation $A X B=C$ over complex field.

\section{Introduction}

Throughout this paper, we denote the set of all $m \times n$ matrices over the real field and complex field by $R^{m \times n}$ and $C^{m \times n}$, respectively. Let the symbols $H C^{n \times n}, S R^{n \times n}, A S R^{n \times n}, I_{n}$, and 0 stand for the set of all $n \times n$ complex Hermitian matrices, the set of all $n \times n$ real symmetric matrices, the set of all $n \times n$ real skew symmetric matrices, the $n \times n$ identity matrix, and the zero matrix with appropriate size, respectively. We also denote the transpose, the conjugate transpose, the Moore-Penrose inverse, and the Frobenius norm of $A$ by $A^{T}, A^{H}, A^{\dagger}$, and $\|A\|$, respectively. Let $A \in C^{m \times n}$; we define $\operatorname{vec}(A)=\left(a_{1}{ }^{T}, a_{2}{ }^{T}, \ldots, a_{n}{ }^{T}\right)^{T}$, where $a_{i}$ is the $i$-column of $A, i=1,2, \ldots, n$.

In this paper, we consider the Hermitian (anti)reflexive solution problem of matrix equation $A X B=C$. Let us first recall the definition of those two kinds of matrices.

Definition 1. Given a generalized reflection matrix $J$, that is, $J^{H}=J, J^{2}=I$, a matrix $A \in C^{n \times n}$ is called a Hermitian reflexive matrix with respect to $J$ if $A^{H}=A$ and $A=J A J$. The set of all $n \times n$ Hermitian reflexive matrices with respect to $J$ is denoted by $H C_{r}^{n \times n}(J)$.
Definition 2. Given a generalized reflection matrix $J$, that is, $J^{H}=J, J^{2}=I$, a matrix $A \in C^{n \times n}$ is called a Hermitian antireflexive matrix with respect to $J$ if $A^{H}=A$ and $A=-J A J$. The set of all $n \times n$ Hermitian antireflexive matrices with respect to $J$ is denoted by $H C_{a}^{n \times n}(J)$.

As an important kind of matrices, the reflexive matrices with different constraints were studied by many authors in recent years in literatures and references therein [1-29]. For instance, Zhao et al. [1] derived the general Hermitian reflexive solution to $A X=B$, while Yu and Shen [2] considered a more general case, that is, the Hermitian $\{J, k+1\}$-(anti)reflexive solution to $A X=B$. As an extension of matrix equation $A X=B$, the matrix system $A X=B, X C=D$ was considered by Zhou and Yang [3]. They gave the necessary and sufficient conditions for the existence of a Hermitian reflexive solution to the above matrix system. As we can see, many important associated works were widely studied. But finding the least squares Hermitian (anti)reflexive solution with the least norm to matrix equation $A X B=C$ is still a problem. Therefore, we in this paper will fill this gap.

We consider the following two problems. 
Problem 3. Given matrices $A \in C^{m \times n}, B \in C^{n \times k}$, and $C \in$ $C^{m \times k}$, let

$$
\begin{aligned}
S & =\left\{X \mid X \in H C_{r}^{n \times n}(J),\|A X B-C\|\right. \\
& \left.=\min _{\widehat{X} \in H C_{r}^{n \times n}(J)}\|A \widehat{X} B-C\|\right\} .
\end{aligned}
$$

Find $X_{0} \in S$ such that $\left\|X_{0}\right\|=\min _{X \in S}\|X\|$.

Problem 4. Given matrices $A \in C^{m \times n}, B \in C^{n \times k}$, and $C \in$ $C^{m \times k}$, let

$$
\begin{aligned}
S_{A} & =\left\{X \mid X \in H C_{a}^{n \times n}(J),\|A X B-C\|\right. \\
& \left.=\min _{\widehat{X} \in H C_{a}^{n \times n}(J)}\|A \widehat{X} B-C\|\right\} .
\end{aligned}
$$

Find $X_{0} \in S_{A}$ such that $\left\|X_{0}\right\|=\min _{X \in S_{A}}\|X\|$.

Now, we start with some useful and fundamental results in the following.

Lemma 5. The least squares solution to the matrix equation $A x=b$ with $A \in C^{m \times n}$ and $b \in C^{m}$ is given by $x=A^{\dagger} b+$ $\left(I-A^{\dagger} A\right) y$, where $y \in C^{n}$ is an arbitrary vector. And the least squares solution with the least norm is $x=A^{\dagger} b$.

Kronecker product method plays an important role in this paper. We use this method to get some important vectorizing formulas.

For any $A \in C^{m \times n}, A=A_{1}+A_{2} i$, where $A_{1}, A_{2} \in R^{m \times n}$. The real representation matrix of $A=A_{1}+A_{2} i \in C^{m \times n}$ is given by $f(A)=\left[\begin{array}{cc}A_{1} & A_{2} \\ -A_{2} & A_{1}\end{array}\right] \in C^{2 m \times 2 n}$, which has the following properties:

(1) $f(A+B)=f(A)+f(B)$.

(2) $f(A B)=f(A) f(B)$.

(3) For $A=A_{1}+A_{2} i \in C^{m \times n}$, we define $g(A)=\left(A_{1}, A_{2}\right)$. Then

$$
\begin{aligned}
\|\operatorname{vec}(A)\| & =\|\operatorname{vec}(g(A))\|=\left\|\left[\begin{array}{c}
\operatorname{vec}\left(A_{1}\right) \\
\operatorname{vec}\left(A_{2}\right)
\end{array}\right]\right\|, \\
g(A B) & =g(A) f(B) .
\end{aligned}
$$

Analogously to the method of Yuan et al. (see [30]), we can get a fundamental result as below.

Lemma 6. Let $A, B$, and $C$ be complex matrices with appropriate sizes; then $\operatorname{vec}(g(A B C))=f\left(C^{H} \otimes \bar{A}\right) \operatorname{vec}(g(B))$.
Proof. We denote $A=A_{1}+A_{2} i, B=B_{1}+B_{2} i$, and $C=$ $C_{1}+C_{2} i . g(A B C)=g(A) f(B C)=g(A) f(B) f(C)$. Then

$$
\begin{aligned}
g & (A) f(B) f(C)=\left[A_{1}, A_{2}\right]\left[\begin{array}{cc}
B_{1} & B_{2} \\
-B_{2} & B_{1}
\end{array}\right]\left[\begin{array}{cc}
C_{1} & C_{2} \\
-C_{2} & C_{1}
\end{array}\right] \\
& =\left[A_{1} B_{1} C_{1}-A_{2} B_{2} C_{1}-\mathrm{A}_{1} B_{2} C_{2}\right. \\
& -A_{2} B_{1} C_{2}, A_{1} B_{1} C_{2}-A_{2} B_{2} C_{2}+A_{1} B_{2} C_{1} \\
& \left.+A_{2} B_{1} C_{1}\right] .
\end{aligned}
$$

By the rule of

$$
\operatorname{vec}(X Y Z)=\left(Z^{T} \otimes X\right) \operatorname{vec}(Y),
$$

we have

$$
\begin{aligned}
& \operatorname{vec}(g(A B C)) \\
& =\left[\begin{array}{ll}
C_{1}^{T} \otimes A_{1}-C_{2}^{T} \otimes A_{2} & -C_{2}^{T} \otimes A_{1}-C_{1}^{T} \otimes A_{2} \\
C_{2}^{T} \otimes A_{1}+C_{1}^{T} \otimes A_{2} & C_{1}^{T} \otimes A_{1}-C_{2}^{T} \otimes A_{2}
\end{array}\right]\left[\begin{array}{l}
\operatorname{vec}\left(B_{1}\right) \\
\operatorname{vec}\left(B_{2}\right)
\end{array}\right] \\
& =f\left(C^{H} \otimes \bar{A}\right)\left[\begin{array}{l}
\operatorname{vec}\left(B_{1}\right) \\
\operatorname{vec}\left(B_{2}\right)
\end{array}\right]=f\left(C^{H} \otimes \bar{A}\right) \operatorname{vec}(g(B)) .
\end{aligned}
$$

The structure of Hermitian (anti)reflexive matrix promises the following decompositions, which would simplify our problems.

Lemma 7. Let $J$ be the $n \times n$ generalized reflection matrix. Then there exists an $n \times n$ unitary matrix $U$ such that

$$
J=U\left[\begin{array}{cc}
I_{r} & 0 \\
0 & -I_{n-r}
\end{array}\right] U^{H} .
$$

Lemma 8 (see [18]). (1) Let $A \in C^{n \times n}$ and let the spectral decomposition of the $n \times n$ reflection matrix $J$ be given as (7). Then $A \in H C_{r}^{n \times n}(J)$ if and only if

$$
A=U\left[\begin{array}{cc}
F_{1} & 0 \\
0 & F_{2}
\end{array}\right] U^{H},
$$

where $F_{1} \in H C^{r \times r}$ and $F_{2} \in H C^{(n-r) \times(n-r)}$.

(2) See [4]. Let $A \in C^{n \times n}$ and let the spectral decomposition of the $n \times n$ reflection matrix $J$ be given as (7). Then $A \in$ $H C_{a}^{n \times n}(J)$ if and only if

$$
A=U\left[\begin{array}{cc}
0 & F \\
F^{H} & 0
\end{array}\right] U^{H},
$$

where $F \in C^{r \times(n-r)}$

\section{The Solution of Problem 3}

In this section, we provide the least squares Hermitian reflexive solution with the least norm to matrix equation $A X B=C$. There are the vectorizing formulas of Hermitian matrix, which are very useful in what comes below. 
Definition 9. Let $X=\left(x_{i j}\right) \in R^{n \times n}$; we define

$$
\operatorname{vec}_{S}(X)=\left(x_{1}, x_{2}, \ldots, x_{n-1}, x_{n}\right)^{T} \in R^{n(n+1) / 2},
$$

where $x_{1}=\left(x_{11}, \sqrt{2} x_{21}, \ldots, \sqrt{2} x_{n 1}\right), x_{2}=\left(x_{22}, \sqrt{2} x_{32}, \ldots\right.$, $\left.\sqrt{2} x_{n 2}\right), \ldots, x_{n-1}=\left(x_{(n-1)(n-1)}, \sqrt{2} x_{n(n-1)}\right)$, and $x_{n}=x_{n n}$.
Definition 10. Let $X=\left(x_{i j}\right) \in R^{n \times n}$; we define

$$
\operatorname{vec}_{A}(X)=\sqrt{2}\left(x_{1}, x_{2}, \ldots, x_{n-2}, x_{n-1}\right)^{T} \in R^{n(n-1) / 2},
$$

where $x_{1}=\left(x_{21}, x_{31}, \ldots, x_{n 1}\right), x_{2}=\left(x_{32}, x_{42}, \ldots, x_{n 2}\right), \ldots$, $x_{n-2}=\left(x_{(n-1)(n-2)}, x_{n(n-2)}\right)$, and $x_{n-1}=x_{n(n-1)}$.

Lemma 11 (see [30]). Suppose that $X \in R^{n \times n}$. Then $X \in S R^{n \times n}$ if and only if $\operatorname{vec}(X)=K_{S} \operatorname{vec}_{S}(X)$, where $\operatorname{vec}_{S}(X)$ is given by (10), where

$$
K_{S}=\frac{1}{\sqrt{2}}\left[\begin{array}{ccccccccccccccc}
\sqrt{2} e_{1} & e_{2} & e_{3} & \cdots & e_{n-1} & e_{n} & 0 & 0 & \cdots & 0 & 0 & \cdots & 0 & 0 & 0 \\
0 & e_{1} & 0 & \cdots & 0 & 0 & \sqrt{2} e_{2} & e_{3} & \cdots & e_{n-1} & e_{n} & \cdots & 0 & 0 & 0 \\
0 & 0 & e_{1} & \cdots & 0 & 0 & 0 & e_{2} & \cdots & 0 & 0 & \cdots & 0 & 0 & 0 \\
\vdots & \vdots & \vdots & & \vdots & \vdots & \vdots & \vdots & & \vdots & \vdots & & \vdots & \vdots & \vdots \\
0 & 0 & 0 & \cdots & e_{1} & 0 & 0 & 0 & \cdots & e_{2} & 0 & \cdots & \sqrt{2} e_{n-1} & e_{n} & 0 \\
0 & 0 & 0 & \cdots & 0 & e_{1} & 0 & 0 & \cdots & 0 & e_{2} & \cdots & 0 & e_{n-1} & \sqrt{2} e_{n}
\end{array}\right] .
$$

Obviously, $e_{i}$ is the i-column of $I_{n}, K_{S} \in R^{n^{2} \times n(n+1) / 2}$, and $K_{S}^{T} K_{S}=I_{n(n+1) / 2}$.

Lemma 12. Suppose that $X \in R^{n \times n}$. Then $X \in A S R^{n \times n}$ if and only if $\operatorname{vec}(X)=K_{A} \operatorname{vec}_{A}(X)$, where $\operatorname{vec}_{A}(X)$ is given by (11), where

$$
\begin{aligned}
K_{A} & =\frac{1}{\sqrt{2}}\left[\begin{array}{ccccccccccc}
e_{2} & e_{3} & \cdots & e_{n-1} & e_{n} & 0 & \cdots & 0 & 0 & \cdots & 0 \\
-e_{1} & 0 & \cdots & 0 & 0 & e_{3} & \cdots & e_{n-1} & e_{n} & \cdots & 0 \\
0 & -e_{1} & \cdots & 0 & 0 & -e_{2} & \cdots & 0 & 0 & \cdots & 0 \\
\vdots & \vdots & & \vdots & \vdots & \vdots & & 0 & 0 & & 0 \\
0 & 0 & \cdots & -e_{1} & 0 & 0 & \cdots & -e_{2} & 0 & \cdots & e_{n} \\
0 & 0 & \cdots & 0 & -e_{1} & 0 & \cdots & 0 & -e_{2} & \cdots & -e_{n-1}
\end{array}\right] .
\end{aligned}
$$

Obviously, $e_{i}$ is the $i$-column of $I_{n}, K_{A} \in R^{n^{2} \times n(n-1) / 2}$, and $K_{A}^{T} K_{A}=I_{n(n-1) / 2}$.

Let $X=X_{1}+X_{2} i \in H C^{n \times n}, X_{1} \in R^{n \times n}, X_{2} \in R^{n \times n}$. Then $X_{1}=X_{1}^{T}$ and $X_{2}=-X_{2}^{T}$. By Lemmas 11 and 12 , one has

$$
\left[\begin{array}{c}
\operatorname{vec}\left(X_{1}\right) \\
\operatorname{vec}\left(X_{2}\right)
\end{array}\right]=\left[\begin{array}{cc}
K_{S} & 0 \\
0 & K_{A}
\end{array}\right]\left[\begin{array}{c}
\operatorname{vec}_{S}\left(X_{1}\right) \\
\operatorname{vec}_{A}\left(X_{2}\right)
\end{array}\right] .
$$

If $X \in H C_{r}^{n \times n}(J)$, then, by Lemma 8 , there exists a unitary matrix $U$ such that

$$
X=U\left[\begin{array}{ll}
Y & 0 \\
0 & Z
\end{array}\right] U^{H}
$$

where $Y=Y_{1}+Y_{2} i \in H C^{r \times r}$, and $Z=Z_{1}+Z_{2} i \in H C^{(n-r) \times(n-r)}$.
Set

$$
\begin{aligned}
A U & =[D, E], \\
U^{H} B=\left[\begin{array}{l}
F \\
G
\end{array}\right], & \\
M & =\left[f\left(F^{H} \otimes \bar{D}\right), f\left(G^{H} \otimes \bar{E}\right)\right] \\
\cdot & {\left[\begin{array}{cccc}
K_{S 1} & 0 & 0 & 0 \\
0 & K_{A 1} & 0 & 0 \\
0 & 0 & K_{S 2} & 0 \\
0 & 0 & 0 & K_{A 2}
\end{array}\right], }
\end{aligned}
$$

where $U$ is given by (7). $K_{S 1}, K_{S 2}$ are given by (12) with the sizes $r^{2} \times r(r+1) / 2,(n-r)^{2} \times(n-r)(n-r+1) / 2$, respectively. $K_{A 1}, K_{A 2}$ are given by (13) with the sizes $r^{2} \times r(r-1) / 2$, $(n-$ $r)^{2} \times(n-r)(n-r-1) / 2$, respectively. Then one has the following result.

Theorem 13. Let $A \in C^{m \times n}, B \in C^{n \times k}$, and $C \in C^{m \times k}$. Then the solution $X \in S$ to matrix equation $A X B=C$ is given by

$$
\begin{aligned}
X & =U\left[\begin{array}{ll}
Y & 0 \\
0 & Z
\end{array}\right] U^{H}, \\
\operatorname{vec}(Y) & =\left[K_{S 1}, K_{A 1} i, 0,0\right]\left[M^{\dagger} c+\left(I-M^{\dagger} M\right) y\right], \\
\operatorname{vec}(Z) & =\left[0,0, K_{S 2}, K_{A 2} i\right]\left[M^{\dagger} c+\left(I-M^{\dagger} M\right) y\right],
\end{aligned}
$$

where $U$ is given by (7), $c=\operatorname{vec}(g(C)) \in R^{2 k m}$, and $y \in$ $R^{r^{2}+(n-r)^{2}}$ is an arbitrary vector. And the solution $X \in S$ with 
the least norm is given by

$$
\begin{aligned}
X & =U\left[\begin{array}{cc}
Y_{0} & 0 \\
0 & Z_{0}
\end{array}\right] U^{H}, \\
\operatorname{vec}\left(Y_{0}\right) & =\left[K_{S 1}, K_{A 1} i, 0,0\right] M^{\dagger} c, \\
\operatorname{vec}\left(Z_{0}\right) & =\left[0,0, K_{S 2}, K_{A 2} i\right] M^{\dagger} c .
\end{aligned}
$$

Proof. Since

$$
\begin{aligned}
& Y_{1} \in S R^{r \times r}, \\
& Y_{2} \in A S R^{r \times r}, \\
& Z_{1} \in S R^{(n-r) \times(n-r),} \\
& Z_{2} \in A S R^{(n-r) \times(n-r),},
\end{aligned}
$$

it follows from (14) that

$$
\left[\begin{array}{c}
\operatorname{vec}\left(Y_{1}\right) \\
\operatorname{vec}\left(Y_{2}\right) \\
\operatorname{vec}\left(Z_{1}\right) \\
\operatorname{vec}\left(Z_{2}\right)
\end{array}\right]=\left[\begin{array}{cccc}
K_{S 1} & 0 & 0 & 0 \\
0 & K_{A 1} & 0 & 0 \\
0 & 0 & K_{S 2} & 0 \\
0 & 0 & 0 & K_{A 2}
\end{array}\right]\left[\begin{array}{c}
\operatorname{vec}_{S}\left(Y_{1}\right) \\
\operatorname{vec}_{A}\left(Y_{2}\right) \\
\operatorname{vec}_{S}\left(Z_{1}\right) \\
\operatorname{vec}_{A}\left(Z_{2}\right)
\end{array}\right] .
$$

Now, by Lemmas 6 and 11 and (23), we have

$$
\begin{aligned}
& \|A X B-C\|=\left\|A U\left[\begin{array}{ll}
Y & 0 \\
0 & Z
\end{array}\right] U^{H} B-C\right\|=\| D Y F \\
& +E Z G-C\|=\| g(D Y F)+g(E Z G)-g(C) \| \\
& =\|\operatorname{vec}(g(D Y F))+\operatorname{vec}(g(E Z G))-\operatorname{vec}(g(C))\| \\
& =\| f\left(F^{H} \otimes \bar{D}\right)\left[\begin{array}{l}
\operatorname{vec}\left(Y_{1}\right) \\
\operatorname{vec}\left(Y_{2}\right)
\end{array}\right] \\
& +f\left(G^{H} \otimes \bar{E}\right)\left[\begin{array}{c}
\operatorname{vec}\left(Z_{1}\right) \\
\operatorname{vec}\left(Z_{2}\right)
\end{array}\right]-\operatorname{vec}(g(C)) \| \\
& =\|\left[f\left(F^{H} \otimes \bar{D}\right), f\left(G^{H} \otimes \bar{E}\right)\right]\left[\begin{array}{c}
\operatorname{vec}\left(Y_{1}\right) \\
\operatorname{vec}\left(Y_{2}\right) \\
\operatorname{vec}\left(Z_{1}\right) \\
\operatorname{vec}\left(Z_{2}\right)
\end{array}\right] \\
& -\operatorname{vec}(g(C))\|=\| M x-c \|,
\end{aligned}
$$

where $M$ is given by (17); $x=\left[\begin{array}{c}\operatorname{vec}_{S}\left(Y_{1}\right) \\ \operatorname{vec}_{A}\left(Y_{2}\right) \\ \operatorname{vec}_{S}\left(Z_{1}\right) \\ \operatorname{vec}_{A}\left(Z_{2}\right)\end{array}\right]$. Then, by Lemma 5,

$$
x=M^{\dagger} c+\left(I-M^{\dagger} M\right) y
$$

is the required least squares solution, where $y \in R^{r^{2}+(n-r)^{2}}$ is an arbitrary vector. Recall that

$$
\left[\begin{array}{c}
\operatorname{vec}\left(Y_{1}\right) \\
\operatorname{vec}\left(Y_{2}\right) \\
\operatorname{vec}\left(Z_{1}\right) \\
\operatorname{vec}\left(Z_{2}\right)
\end{array}\right]=\left[\begin{array}{cccc}
K_{S 1} & 0 & 0 & 0 \\
0 & K_{A 1} & 0 & 0 \\
0 & 0 & K_{S 2} & 0 \\
0 & 0 & 0 & K_{A 2}
\end{array}\right] x
$$

Therefore

$$
\begin{aligned}
\operatorname{vec}(Y) & =\operatorname{vec}\left(Y_{1}\right)+\operatorname{vec}\left(Y_{2}\right) i=\left[\begin{array}{llll}
K_{S 1} & K_{A 1} i & 0 & 0
\end{array}\right] x, \\
\operatorname{vec}(Z) & =\operatorname{vec}\left(Z_{1}\right)+\operatorname{vec}\left(Z_{2}\right) i \\
& =\left[\begin{array}{llll}
0 & 0 & K_{S 2} & K_{A 2} i
\end{array}\right] x .
\end{aligned}
$$

Then the least squares Hermitian reflexive solution is given by (18). Obviously, the least squares Hermitian reflexive solution with the least norm is given by (20).

\section{The Solution of Problem 4}

In this section, we provide the least squares Hermitian antireflexive solution with the least norm to matrix equation $A X B=C$.

We first derive the relation between $\operatorname{vec}(g(Y))$ and $\operatorname{vec}\left(g\left(Y^{H}\right)\right)$.

Lemma 14 (see [31]). Let $Y \in R^{m \times n}$ be any matrix. Then

$$
\operatorname{vec}\left(Y^{T}\right)=P_{(m, n)} \operatorname{vec}(Y),
$$

where

$$
P_{(m, n)}=\left[\begin{array}{cccc}
E_{11}^{T} & E_{12}^{T} & \cdots & E_{1 n}^{T} \\
E_{21}^{T} & E_{22}^{T} & \cdots & E_{2 n}^{T} \\
\vdots & \vdots & \ddots & \vdots \\
E_{m 1}^{T} & E_{m 2}^{T} & \cdots & E_{m n}^{T}
\end{array}\right] \in R^{m n \times m n}
$$

with $E_{i j}, i=1,2, \ldots, m, j=1,2, \ldots, n$, being an $m \times n$ matrix with the element at position $(i, j)$ being 1 and the others being 0 . Moreover, $P_{(m, n)}$ is an orthogonal matrix, which is uniquely determined by the integers $m, n$.

Let $X \in H C_{a}^{n \times n}(J)$; then, by Lemma 8, there exists a unitary matrix $U$ such that

$$
X=U\left[\begin{array}{cc}
0 & Y \\
Y^{H} & 0
\end{array}\right] U^{H},
$$

where $Y \in C^{r \times(n-r)}$ and $U$ is given by (7). Since $Y^{H}=Y_{1}^{T}-Y_{2}^{T} i$,

$$
g\left(Y^{H}\right)=\left(Y_{1}^{T},-Y_{2}^{T}\right) \text {. }
$$


By Lemma 14, one has

$$
\begin{aligned}
\operatorname{vec}\left(g\left(Y^{H}\right)\right) & =\left[\begin{array}{c}
\operatorname{vec}\left(Y_{1}^{T}\right) \\
\operatorname{vec}\left(-Y_{2}^{T}\right)
\end{array}\right]=\left[\begin{array}{c}
P_{(r, n-r)} \operatorname{vec}\left(Y_{1}\right) \\
-P_{(r, n-r)} \operatorname{vec}\left(Y_{2}\right)
\end{array}\right] \\
& =\left[\begin{array}{cc}
P_{(r, n-r)} & 0 \\
0 & -P_{(r, n-r)}
\end{array}\right] \operatorname{vec}(g(Y)) .
\end{aligned}
$$

Set

$$
\begin{aligned}
A U= & {[D, E], } \\
U^{H} B= & {\left[\begin{array}{l}
F \\
G
\end{array}\right], } \\
N= & f\left(F^{H} \otimes \bar{E}\right)\left[\begin{array}{cc}
P_{(r, n-r)} & 0 \\
0 & -P_{(r, n-r)}
\end{array}\right] \\
& +f\left(G^{H} \otimes \bar{D}\right),
\end{aligned}
$$

and $P_{(r, n-r)}$ is given by (29). Then one has the following results.

Theorem 15. Let $A \in C^{m \times n}, B \in C^{n \times k}$, and $C \in C^{m \times k}$. Then on has the following:

(1) The solution $X \in S_{A}$ to matrix equation $A X B=C$ is given by

$$
X=U\left[\begin{array}{cc}
0 & Y \\
Y^{H} & 0
\end{array}\right] U^{H},
$$

where

$$
\operatorname{vec}(Y)=\left[I_{r(n-r)}, i I_{r(n-r)}\right]\left[N^{\dagger} c+\left(I-N^{\dagger} N\right) y\right]
$$

$U$ is given by (7), $c=\operatorname{vec}(g(C)) \in R^{2 k m}$, and $y \in R^{2 r(n-r)}$ is an arbitrary vector.

(2) The solution $X \in S_{A}$ with the least norm is given by

$$
X=U\left[\begin{array}{cc}
0 & Y_{0} \\
Y_{0}^{H} & 0
\end{array}\right] U^{H}
$$

where $U$ is given by (7) and $\operatorname{vec}\left(Y_{0}\right)=\left[I_{r(n-r)}, i I_{r(n-r)}\right] N^{\dagger} c$, $c=\operatorname{vec}(g(C))$.

Proof. It follows from Lemma 6 that

$$
\begin{gathered}
\operatorname{vec}\left(g\left(E Y^{H} F\right)\right)=f\left(F^{H} \otimes \bar{E}\right) \operatorname{vec}\left(g\left(Y^{H}\right)\right), \\
\operatorname{vec}(g(D Y G))=f\left(G^{H} \otimes \bar{D}\right) \operatorname{vec}(g(Y)) .
\end{gathered}
$$

Combining (32) and (37), we obtain

$$
\begin{aligned}
& \operatorname{vec}\left(g\left(E Y^{H} F\right)\right)=f\left(F^{H} \otimes \bar{E}\right) \operatorname{vec}\left(g\left(Y^{H}\right)\right) \\
& =f\left(F^{H} \otimes \bar{E}\right)\left[\begin{array}{cc}
P_{(r, n-r)} & 0 \\
0 & -P_{(r, n-r)}
\end{array}\right] \operatorname{vec}(g(Y)) .
\end{aligned}
$$

Then

$$
\begin{aligned}
& \|A X B-C\|=\left\|A U\left[\begin{array}{cc}
0 & Y \\
Y^{H} & 0
\end{array}\right] U^{H} B-C\right\|=\| E Y^{H} F \\
& +D Y G-C\|=\| g\left(E Y^{H} F\right)+g(D Y G)-g(C) \| \\
& =\| \operatorname{vec}\left(g\left(E Y^{H} F\right)\right)+\operatorname{vec}(g(D Y G)) \\
& -\operatorname{vec}(g(C)) \| \\
& =\| f\left(F^{H} \otimes \bar{E}\right)\left[\begin{array}{cc}
P_{(r, n-r)} & 0 \\
0 & -P_{(r, n-r)}
\end{array}\right] \operatorname{vec}(g(Y)) \\
& +f\left(G^{H} \otimes \bar{D}\right) \operatorname{vec}(g(Y))-\operatorname{vec}(g(C))\|=\| N x \\
& -c \|,
\end{aligned}
$$

where $c=\operatorname{vec}(g(C))$ and $x=\operatorname{vec}(g(Y))$.

By Lemma 5, the least squares solution is given by

$$
x=\left[\begin{array}{c}
\operatorname{vec}\left(Y_{1}\right) \\
\operatorname{vec}\left(Y_{2}\right)
\end{array}\right]=N^{\dagger} c+\left(I-N^{\dagger} N\right) y \text {, }
$$

where $y \in R^{2 r(n-r)}$ is an arbitrary vector.

The least squares solution with least norm is $x_{0}=N^{\dagger} c$. So

$$
\begin{aligned}
\operatorname{vec}\left(Y_{1}\right) & =\left[I_{r(n-r)}, 0\right] x, \\
\operatorname{vec}\left(Y_{2}\right) & =\left[0, I_{r(n-r)}\right] x, \\
\operatorname{vec}\left(Y_{10}\right) & =\left[I_{r(n-r)}, 0\right] x_{0}, \\
\operatorname{vec}\left(Y_{20}\right) & =\left[0, I_{r(n-r)}\right] x_{0} .
\end{aligned}
$$

Now, it is easy to see that our required solutions are given by (34) and (36).

\section{Conclusion}

By exploiting the decomposition of Hermitian (anti)reflexive matrix and the Kronecker product, we derive the expression of the least squares Hermitian (anti)reflexive solution with the least norm to $A X B=C$. This method overcomes the difficulty of finding the structured least squares solution with the least norm but creates a large-scale linear system $\widehat{A} x=\widehat{b}$. Therefore, it fits well with the small-scale coefficient matrix, but, for the large-scale case, it may not work so well.

\section{Conflicts of Interest}

The authors declare that they have no conflicts of interest.

\section{Authors' Contributions}

Both authors read and approved the final manuscript. 


\section{Acknowledgments}

This research was supported by Macau Science and Technology Development Fund (no. 003/2015/A1) and a grant from the National Natural Science Foundation of China (11571220).

\section{References}

[1] L. Zhao, X. Hu, and L. Zhang, "Linear restriction problem of Hermitian reflexive matrices and its approximation," Applied Mathematics and Computation, vol. 200, no. 1, pp. 341-351, 2008.

[2] J. Yu and S.-q. Shen, "The Hermitian $\{P, k+1\}$-(anti-)reflexive solutions of a linear matrix equation," Computers \& Mathematics with Applications, vol. 71, no. 12, pp. 2513-2523, 2016.

[3] S. Zhou and S. Yang, "The Hermitian reflexive solutions and the anti-Hermitian reflexive solutions for a class of matrix equations $(A X=B, X C=D)$," Energy Procedia, vol. 17, part B, pp. 1591-1597, 2012.

[4] Z.-Y. Peng, "The inverse eigenvalue problem for Hermitian antireflexive matrices and its approximation," Applied Mathematics and Computation, vol. 162, no. 3, pp. 1377-1389, 2005.

[5] L. Lebtahi, O. Romero, and N. Thome, "Characterizations of $\{K, s+1\}$-potent matrices and applications," Linear Algebra and its Applications, vol. 436, no. 2, pp. 293-306, 2012.

[6] L. Lebtahi and N. Thome, "A note on $k$-generalized projections," Linear Algebra and its Applications, vol. 420, no. 2-3, pp. 572575, 2007.

[7] L. Lebtahi, Ó. Romero, and N. Thome, "Relations between $\{K, s+1\}$-potent matrices and different classes of complex matrices," Linear Algebra and its Applications, vol. 438, no. 4, pp. 1517-1531, 2013.

[8] L. Lebtahi, Ó. Romero, and N. Thome, "Algorithms for $\{K, s+$ 1 -potent matrix constructions," Journal of Computational and Applied Mathematics, vol. 249, pp. 157-162, 2013.

[9] S. Gigola, L. Lebtahi, and N. Thome, "The inverse eigenvalue problem for a Hermitian reflexive matrix and the optimization problem," Journal of Computational and Applied Mathematics, vol. 291, pp. 449-457, 2016.

[10] M. Dehghan and M. Hajarian, "Two algorithms for finding the Hermitian reflexive and skew-Hermitian solutions of Sylvester matrix equations," Applied Mathematics Letters, vol. 24, no. 4, pp. 444-449, 2011.

[11] M. Dehghan and M. Hajarian, "An iterative algorithm for the reflexive solutions of the generalized coupled Sylvester matrix equations and its optimal approximation," Applied Mathematics and Computation, vol. 202, no. 2, pp. 571-588, 2008.

[12] M. Dehghan and M. Hajarian, "On the reflexive and antireflexive solutions of the generalised coupled Sylvester matrix equations," International Journal of Systems Science. Principles and Applications of Systems and Integration, vol. 41, no. 6, pp. 607-625, 2010.

[13] M. Dehghan and M. Hajarian, "Finite iterative algorithms for the reflexive and anti-reflexive solutions of the matrix equation $A_{1} X_{1} B_{1}+A_{2} X_{2} B_{2}=C$," Mathematical and Computer Modelling, vol. 49, no. 9-10, pp. 1937-1959, 2009.

[14] M. Dehghan and M. Hajarian, "On the reflexive solutions of the matrix equation $A X B+C Y D=E$," Bulletin of the Korean Mathematical Society, vol. 46, no. 3, pp. 511-519, 2009.

[15] M. Dehghan and M. Hajarian, "The reflexive and anti-reflexive solutions of a linear matrix equation and systems of matrix equations," The Rocky Mountain Journal of Mathematics, vol. 40, no. 3, pp. 825-848, 2010.

[16] F. L. Li, X. Y. Hu, and L. Zhang, "The generalized reflexive solutions for a class of matrix equations $A X=B, X C=D$," Acta Mathematica Scientia, vol. 28B, no. 1, pp. 185-193, 2008.

[17] F.-L. Li, X.-Y. Hu, and L. Zhang, "The generalized anti-reflexive solutions for a class of matrix equations $(B X=C, X D=E)$," Computational \& Applied Mathematics, vol. 27, no. 1, pp. 31-46, 2008.

[18] Z.-y. Peng and X.-y. Hu, "The reflexive and anti-reflexive solutions of the matrix equation $A X=B$," Linear Algebra and Its Applications, vol. 375, pp. 147-155, 2003.

[19] H.-C. Chen, "Generalized reflexive matrices: special properties and applications," SIAM Journal on Matrix Analysis and Applications, vol. 19, no. 1, pp. 140-153, 1998.

[20] D. S. Cvetković-Iliíc, “The reflexive solutions of the matrix equation $A X B=C$," Computers \& Mathematics with Applications, vol. 51, no. 6-7, pp. 897-902, 2006.

[21] H.-x. Chang and Q.-w. Wang, "Reflexive solution to a system of matrix equations," Journal of Shanghai University, vol. 11, no. 4, pp. 355-358, 2007.

[22] Q. W. Wang and F. Zhang, "The reflexive re-nonnegative definite solution to a quaternion matrix equation," Electronic Journal of Linear Algebra, vol. 17, pp. 88-101, 2008.

[23] X.-y. Peng, X.-y. Hu, and L. Zhang, "The reflexive and antireflexive solutions of the matrix equation $A^{H} X B=C$," Journal of Computational and Applied Mathematics, vol. 200, no. 2, pp. 749-760, 2007.

[24] Z. Y. Peng, Y. B. Deng, and J. W. Liu, "Least-squares solution of inverse problem for Hermitian anti-reflexive matrices and its approximation," Acta Mathematica Sinica (English Series), vol. 22, no. 2, pp. 477-484, 2006.

[25] Z. Peng and H. Xin, "The reflexive least squares solutions of the general coupled matrix equations with a submatrix constraint," Applied Mathematics and Computation, vol. 225, pp. 425-445, 2013.

[26] A. Herrero and N. Thome, "Using the GSVD and the lifting technique to find $\{P, k+1\}$ reflexive and anti-reflexive solutions of $A X B=C$," Applied Mathematics Letters, vol. 24, no. 7, pp. 1130-1141, 2011.

[27] M.-1. Liang, L.-f. Dai, and Y.-f. Yang, "The $\{P, Q, k+1\}$-reflexive solution of matrix equation $A X B=C$," Journal of Applied Mathematics and Computing, vol. 42, no. 1-2, pp. 339-350, 2013.

[28] F. P. Beik and M. M. Moghadam, "The general coupled linear matrix equations with conjugate and transpose unknowns over the mixed groups of generalized reflexive and anti-reflexive matrices," Computational \& Applied Mathematics, vol. 33, no. 3, pp. 795-820, 2014.

[29] J.-C. Zhang, S.-Z. Zhou, and X.-Y. Hu, “The $(P, Q)$ generalized reflexive and anti-reflexive solutions of the matrix equation $A X=B$," Applied Mathematics and Computation, vol. 209, no. 2, pp. 254-258, 2009.

[30] S. Yuan, A. Liao, and Y. Lei, "Least squares Hermitian solution of the matrix equation $(A X B, C X D)=(E, F)$ with the least norm over the skew field of quaternion," Mathematical and Computer Modelling, vol. 48, no. 1-2, pp. 91-100, 2008.

[31] J. L. Chen and X. H. Chen, Special Matrices, Tsinghua University Press, Beijing, China, 2002. 


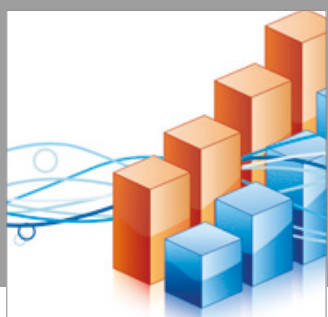

Advances in

Operations Research

vatersals

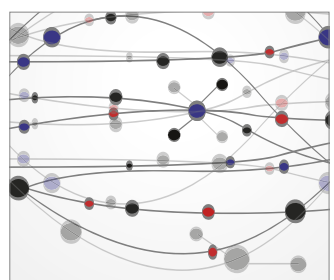

\section{The Scientific} World Journal

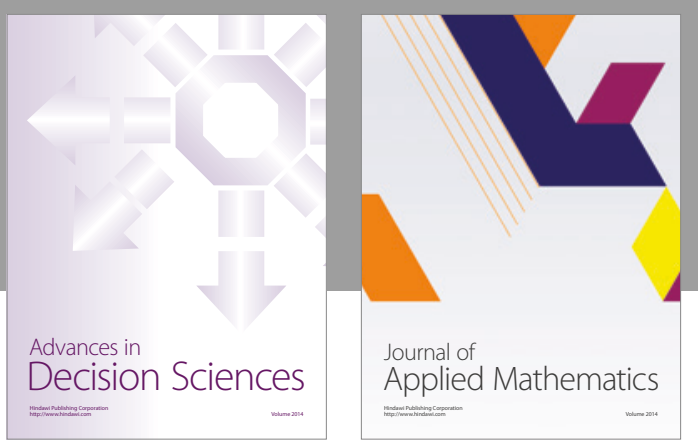

Algebra

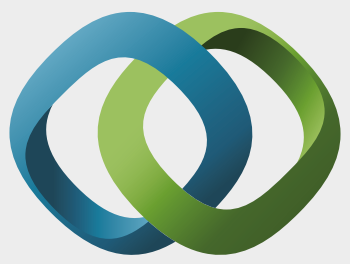

\section{Hindawi}

Submit your manuscripts at

https://www.hindawi.com


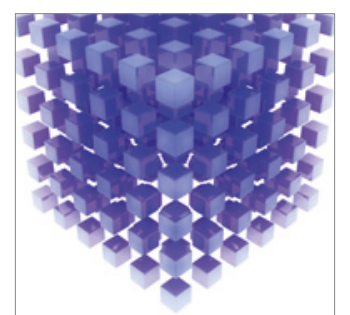

Mathematical Problems in Engineering
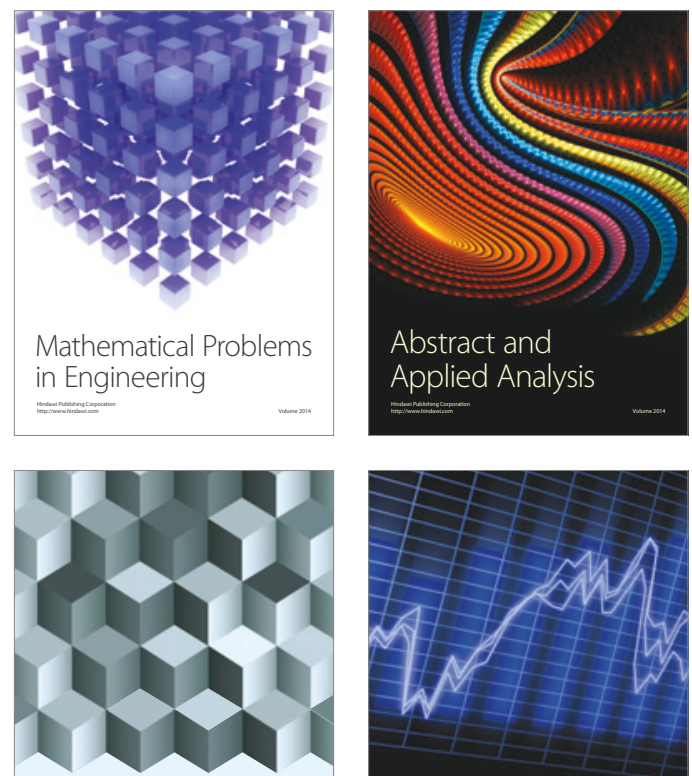

Journal of

Function Spaces

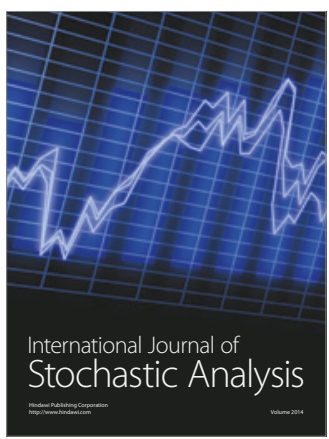

Probability and Statistics
